04

\title{
Источник ускоренного потока металлической плазмы с регулируемыми параметрами
}

\author{
(C) В.Л. Паперный ${ }^{1}$, Н.В. Астраханцев ${ }^{2}$, Н.В. Лебедев ${ }^{2}$ \\ ${ }^{1}$ Иркутский государственный университет, \\ Иркутск, Россия \\ ${ }^{2}$ Иркутский научно-исследовательский технический университет, \\ Иркутск, Россия \\ E-mail: paperny@math.isu.runnet.ru
}

Поступило в Редакцию 7 сентября 2018 г.

В окончательной редакции 24 декабря 2018 г.

Принято к публикации 24 декабря 2018 г.

Исследовались параметры потока металлической плазмы, генерируемого источником с жидким нагреваемым анодом, при движении потока в аксиальном магнитном поле. Показано, что в случае медного рабочего тела источник создает поток плазмы со степенью ионизации около $8 \%$ и концентрацией, вблизи источника достигающей $4 \cdot 10^{16} \mathrm{~m}^{-3}$. Направленная энергия и ширина энергетического спектра ионов потока варьируются в широком диапазоне значений путем изменения напряжений на электродах.

DOI: 10.21883/PJTF.2019.06.47492.17519

Источники ускоренных потоков металлической плазмы широко используются в промышленности, например, для нанесения покрытий, улучшающих эксплуатационные характеристики обрабатываемых изделий. Для этих задач применяются плазменные источники различных видов: вакуумно-дуговые разряды с катодными микропятнами или с диффузной привязкой катодного пятна, стационарные и импульсные магнетронные разряды и др. [1-3]. Характерными особенностями этих разрядов являются относительно невысокая направленная энергия ионов в генерируемом плазменном потоке, в основном находящаяся в диапазоне нескольких десятков электронвольт, и большая ширина энергетического спектра, близкая к величине направленной энергии [4,5]. Существенным недостатком подобных источников также является отсутствие возможности эффективной регулировки этих параметров при заданном материале катода.

В настоящей работе исследовалась возможность создания источника потока металлической плазмы с параметрами ионного энергетического спектра, регулируемыми в широком диапазоне значений. С этой целью использовался несамостоятельный вакуумно-дуговой разряд с испаряемым жидким анодом, нагрев которого до рабочей температуры $T_{a}$ осуществляется внешним источником и/или током дуги [6,7]. Для дуг этого типа характерны высокие значения $T_{a}\left(>10^{3} \mathrm{~K}\right)$, низкие плотности тока разряда $\left(<10^{8} \mathrm{~A} / \mathrm{m}^{2}\right)$, а также возможность управления направленной энергией плазменного потока путем варьирования анодного напряжения, при этом ускорение ионов происходит в электрическом поле между анодом и заземленной стенкой рабочей камеры [8].

Существенным недостатком такого плазменного источника является низкая степень ионизации рабочего тела. Для ее повышения разряд помещают в продольное магнитное поле и используют дополнительный кольце- вой, коаксиальный аноду электрод, на который подается положительный потенциал [9]. Однако измерения показали, что при этом регулируемый диапазон энергий плазменного потока оказывается весьма ограниченным, поскольку вследствие падающей вольт-амперной характеристики разряда увеличение разрядного напряжения с целью повышения энергии ионов приводит к падению тока ниже порового значения [10]. По этой причине данный источник плазмы был существенно модифицирован.

Схема экспериментальной установки приведена на рис. 1. Рабочее вещество помещалось в графитовый тигель диаметром $3 \cdot 10^{-2} \mathrm{~m}$ и высотой $8 \cdot 10^{-3} \mathrm{~m}$. Площадь поверхности расплавленного рабочего вещества составляла $4 \cdot 10^{-4} \mathrm{~m}^{2}$. Накаливаемая заземленная вольфрамовая спираль-катод помещалась на расстоянии $3 \cdot 10^{-3} \mathrm{~m}$ над поверхностью тигля (рис. 1 , вставка $a$ ). Мощность накального источника варьировалась в диапазоне 200-250 W. На тигель-анод подавалось напряжение $U_{a}=+600 \mathrm{~V}$ относительно спирали, в результате чего он разогревался током термоэлектронной эмиссии катода до температуры, при которой начиналось интенсивное испарение атомов рабочего вещества, ионизация последнего в межэлектродном промежутке приводила к возникновению плазмы. Основные измерения параметров плазменного потока были проведены с медью в качестве рабочего вещества.

Источник анодного напряжения с падающей вольтамперной характеристикой обеспечивал в рабочем режиме напряжение горения разряда $U_{a}$ в диапазоне $50-250 \mathrm{~V}$, при этом разрядный ток $I_{d}$ оставался неизменным, равным $3.5 \mathrm{~A}$. В отличие от $[9,10]$ величина $U_{a}$ в рабочем режиме устанавливалась предварительно путем варьирования мощности накального источника.

Над тиглем на расстоянии $10^{-2} \mathrm{~m}$ помещался дополнительный кольцевой электрод высотой $2.5 \cdot 10^{-2} \mathrm{~m}$ 


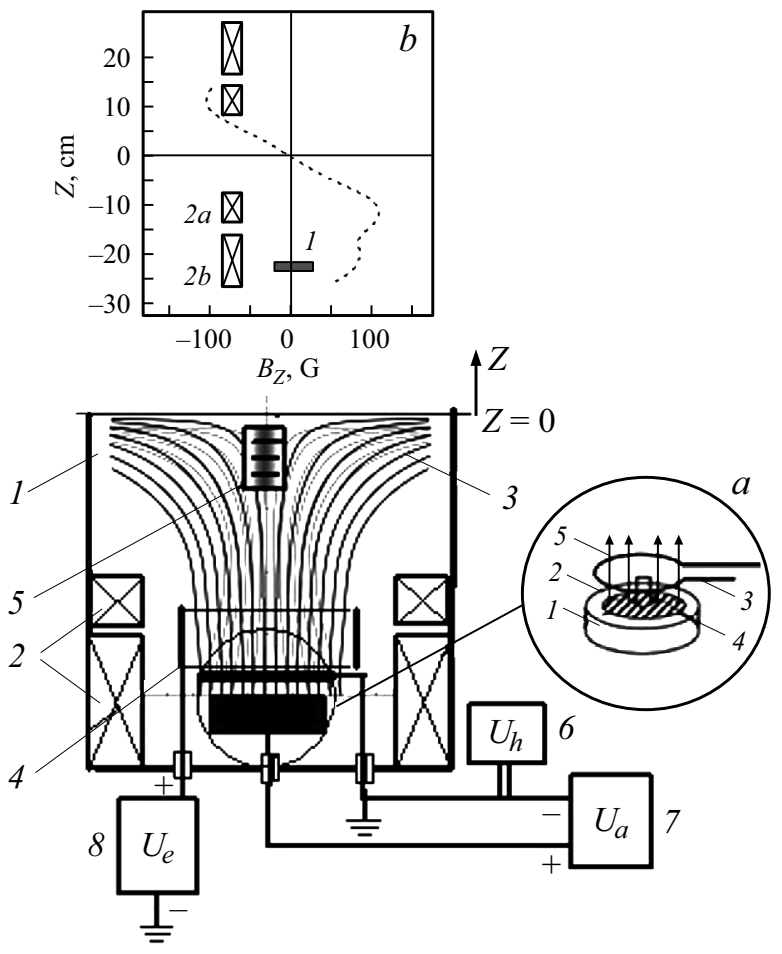

Рис. 1. Схема эксперимента (нижняя половина установки). 1 - рабочая камера, 2 - соленоиды магнитного поля, 3 - рассчитанная карта силовых линий магнитного поля, 4 - дополнительный электрод, 5 - энергоанализатор ионов; источники напряжений: $6-$ накального, $7-$ разрядного, 8 - дополнительного электрода. На вставке $a-$ схема источника плазмы: 1 - тигель-анод, 2 - рабочее вещество, 3 - накаливаемая спираль-катод, $4-$ поток электронов, $5-$ поток атомов; на вставке $b$ - измеренное распределение на оси $Z$ продольной компоненты магнитного поля $B_{Z} .1$ - тигель-анод, $2 a, 2 b-$ соленоиды.

и диаметром $8 \cdot 10^{-2} \mathrm{~m}$, на который подавался потенциал $+U_{e}$, варьируемый в диапазоне 50-110 V. Плазма, проходя через электрод, расширялась в заземленную водоохлаждаемую рабочую камеру, где поддерживалось остаточное давление около $2 \cdot 10^{-3} \mathrm{~Pa}$.

Ведущее магнитное поле с аксиально-симметричной конфигурацией силовых линий создавалось системой двух пар соленоидов. С учетом последующего использования данного источника плазмы для задач плазменной сепарации магнитные силовые линии, пересекающие плоскость тигля, образовывали структуру типа „фонтан“, для чего каждая пара соленоидов включалась последовательно, а их токи были направлены навстречу (рис. 1). Как видно из рисунка, такая конфигурация магнитного поля и электродной системы в отличие от ранее использованной геометрии $[9,10]$ обеспечивала формирование протяженной области квазиоднородного магнитного поля, где двигался генерируемый источником плазменный поток. Величина продольной компоненты магнитного поля $B_{Z}$ на оси установки в этой области при типичных токах соленоидов $2 a$ и $2 b$, равных 2 и 0.5 А соответственно, находилась в диапазоне
$(7-10) \cdot 10^{-3}$ Т и обращалась в нуль в среднем сечении вакуумного объема (рис. 1, вставка $b$ ).

Температура рабочего вещества $T_{a}$ определялась по спектру теплового излучения, измеренному с помощью волоконного спектрометра AvaSpec-2048 (не показан на рис. 1) с использованием методики, описанной в [11], и равнялась $1410 \mathrm{~K}$. Спектральные измерения показали также, что в спектре излучения плазменной струи отсутствуют линии ионов меди, что свидетельствует о невысокой степени ионизации плазмы и позволяет предположить наличие в ней лишь однозарядных ионов.

Энергетический спектр ионов измерялся с помощью трехсеточного анализатора с тормозящим электрическим полем, находящегося на расстоянии $0.15 \mathrm{~m}$ от тигля в области квазиоднородного магнитного поля.

По ионному току насыщения плоского ленгмюровского зонда площадью $2 \cdot 10^{-5} \mathrm{~m}^{2}$ (не показан на рис. 1 ), ориентированного перпендикулярно потоку плазмы и расположенного на расстоянии $0.12 \mathrm{~m}$ от анода, измерялась концентрация ионов. В предположении формирования слоя объемного заряда вблизи зонда оценка дает значение $4 \cdot 10^{16} \mathrm{~m}^{-3}$ на оси камеры при типичных параметрах разряда: $I_{d}=3.5 \mathrm{~A}, U_{a}=200 \mathrm{~V}, U_{e}=110 \mathrm{~V}$.

Весовым методом также определялась степень ионизации плазменной струи, которая оказалась равной $\eta \approx 8 \%$ при указанных параметрах разряда. Степень ионизации рассчитывалась согласно „классическому“ соотношению $\eta=n_{i} /\left(n_{i}+n_{a}\right)$, где $n_{i}$ и $n_{a}-$ концентрации ионов и нейтральных атомов соответственно. Полученная величина характеризует эффективность источника, определяя долю испаренных атомов, переведенных в плазменную фазу. Заметим, что в ряде предыдущих работ [8-10] степень ионизации определялась непосредственно как величина отношения потоков ионов и атомов на зонд, т. е. указанный выше параметр $\eta$ фактически умножался на отношение направленной скорости ионов и тепловой

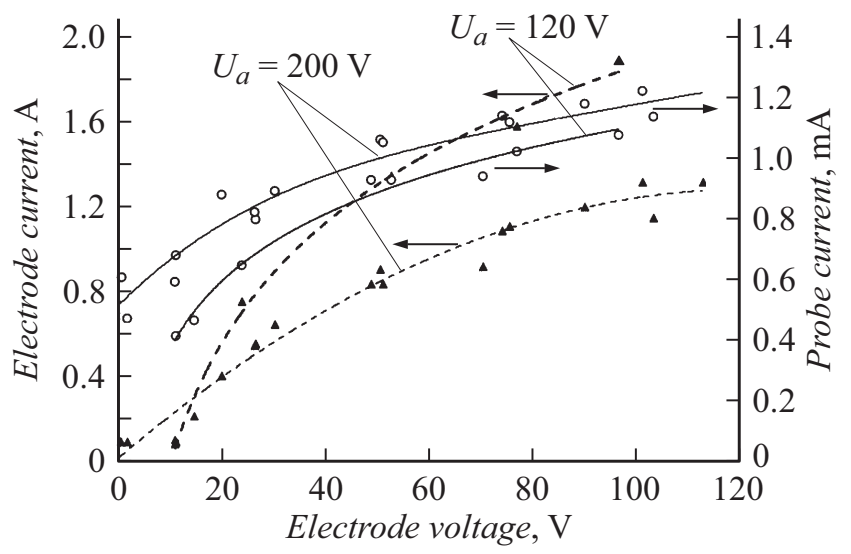

Рис. 2. Зависимость ионного тока насыщения ленгмюровского зонда (сплошные кривые) и тока на электрод 4 (штриховые кривые) от напряжения дополнительного электрода при различных анодных напряжениях. Для наглядности приведена абсолютная величина электронного тока на электрод. 

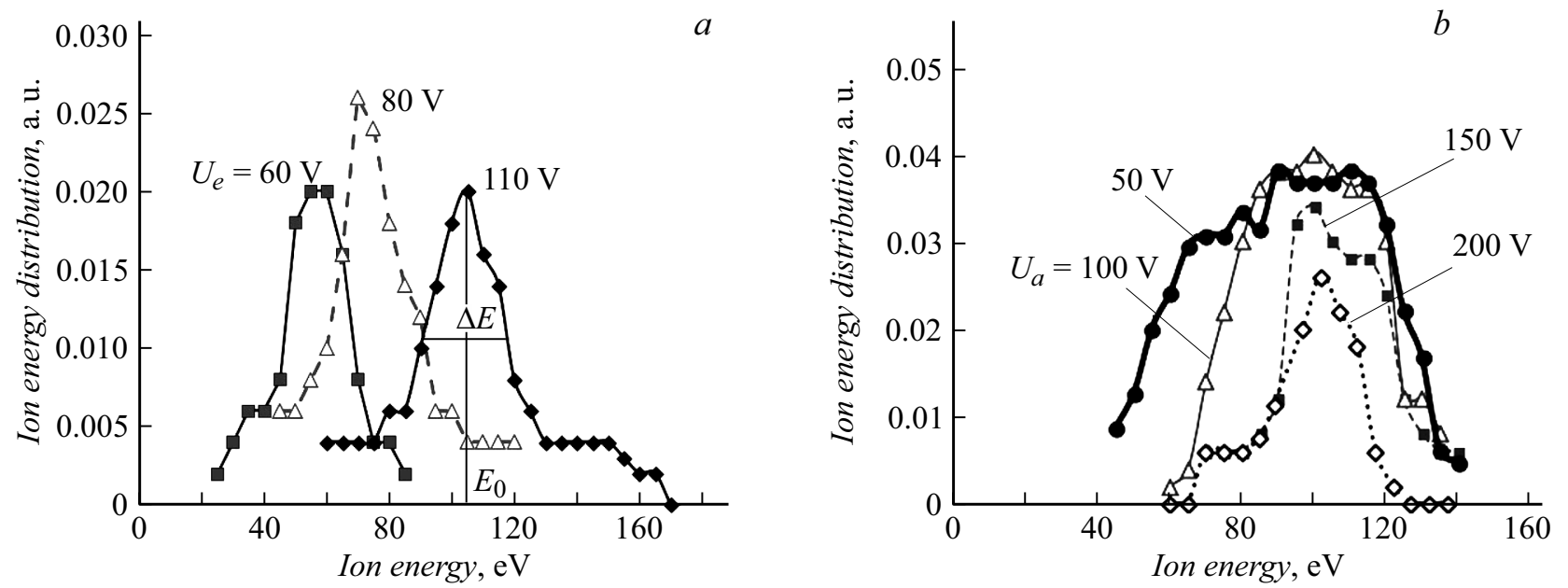

Рис. 3. Энергетический спектр ионов плазменного потока. $a-$ при фиксированном анодном напряжении $U_{a}=200 \mathrm{~V}$ и различных напряжениях дополнительного электрода; $b-$ при фиксированном напряжении дополнительного электрода $U_{e}=105 \mathrm{~V}$ и различных анодных напряжениях.

скорости атомов, лежащее в диапазоне 3-10 в данных экспериментальных условиях.

Влияние дополнительного электрода на эффективность ионизации плазменного потока иллюстрирует рис. 2. Из рисунка следует, что ток на электрод быстро растет с увеличением напряжения, при этом наблюдается также рост тока зонда приблизительно в 2.5 раза с увеличением $U_{e}$ от нуля до $110 \mathrm{~V}$. В указанном предположении формирования призондового слоя ток зонда пропорционален концентрации ионов, что позволяет предполагать такой же рост степени ионизации плазмы. Таким образом, можно заключить, что введение дополнительного электрода в сочетании с наложенным на разряд магнитным полем приводит к существенному росту концентрации плазмы.

Для выяснения возможности управления параметрами ионной компоненты плазменного потока были проведены измерения энергетического спектра ионов при разных напряжениях на электродах. Из рис. 3, a, где приведены результаты измерений, видно, что при увеличении $U_{e}$ от 60 до $110 \mathrm{~V}$ при неизменном напряжении анода $U_{a}$ направленная энергия ионов $E_{0}$ также возрастает от 60 до $112 \mathrm{eV}$, при этом относительная ширина спектра $\Delta E / E_{0}$ составляет менее $30 \%$.

Величина $\Delta E$ существенно зависит от анодного напряжения $U_{a}$. Так, уменьшение $U_{a}$ с 200 до $50 \mathrm{~V}$ при фиксированном напряжении $U_{e} \approx 100 \mathrm{~V}$ приводит к росту ширины спектра более чем в 3 раза (рис. $3, b$ ). Зондовые измерения плавающего потенциала на расстоянии $0.2 \mathrm{~m}$ от анода показали, что он слабо зависит от параметров разряда и лежит вблизи нуля. С учетом того, что температура электронов в данном типе разряда составляет около $5 \mathrm{eV}$ [9], а в разряде, по-видимому, присутствуют лишь однозарядные ионы, это означает, что приведенные выше значения направленной энергии ионов могут быть завышены на $\sim 20 \mathrm{eV}$.
Особенностью данного источника плазменного потока является сохранение неизменным разрядного тока при варьировании разрядного напряжения, которое осуществляется независимым образом путем изменения мощности нагрева анода (т.е. потока испаряемых атомов) внешним источником. Это позволяет предположить, что увеличение мощности разряда вследствие увеличения анодного напряжения расходуется в основном на дополнительную ионизацию атомов рабочего вещества, а не на ускорение плазменного потока.

Согласно оценкам, при данных параметрах плазмы и величине магнитного поля электроны являются замагниченными, а ионы - незамагниченными. Поэтому можно полагать, что рост концентрации плазмы при увеличении напряжения $U_{e}$ связан с повышением эффективности процесса ионизации вследствие возрастания азимутального дрейфового тока замагниченных электронов в скрещенных полях: аксиальном магнитном и радиальном электрическом, которое формируется в плазменной струе при подаче на электрод 4 напряжения $U_{e}$. Вследствие этого образующаяся в области электрода плазма в основном приобретает его потенциал. При последующем движении плазменного потока в протяженной области квазиоднородного магнитного поля, где плазма удерживается благодаря замагниченным электронам, поток ускоряется продольной компонентой электрического поля этого электрода. Таким образом удается разделить цепи питания разрядного и ускорительного промежутков, так что в отличие от предыдущих исследований $[9,10]$ увеличение $U_{e}$ и соответственно энергии ионов не приводит к изменению характеристик разряда, а достигаемая энергия определяется лишь характеристиками источника ускоряющего напряжения $U_{e}$. При этом в принципе можно получить более высокие значения энергии, чем в подобных источниках плазмы $[8,10]$, 
а также независимым образом регулировать ширину энергетического спектра ионов плазменной струи.

Таким образом, в работе исследован источник потока металлической плазмы с жидким испаряемым анодом, в котором управление направленной энергией $E_{0}$ и шириной энергетического спектра ионов $\Delta E$ в широких пределах осуществляется независимым способом путем изменения напряжений на электродной системе.

Результаты частично получены с использованием оборудования ЦКП „Байкальский центр нанотехнологий“. Работа выполнена при поддержке РФФИ (проект № 17-02-00572).

\section{Список литературы}

[1] Anders A. Cathodic arcs: from fractal spots to energetic condensation. N. Y.: Springer, 2008. 543 p.

[2] Paperny V.L., Krasov V.I., Lebedev N., Astrakchantsev N.V., Chernich A.A. // Plasma Sources Sci. Technol. 2014. V. 24. N 1. P. 015009.

[3] Oks E., Anders A. // J. Appl. Phys. 2009. V. 105. N 9. P. 093304.

[4] Paperny V.L., Chernich A.A., Astrakchantsev N.V., Lebedev N.V. // J. Phys. D: Appl. Phys. 2009. V. 42. N 15. P. 155201.

[5] Соловьев А.А., Сочугов Н.С., Оскомов К.В., Работкин С.В. // Физика плазмы. 2009. Т. 35. № 5. С. 443-452.

[6] Дороднов А.М., Кузнещов А.Н., Петросов В.А. // Письма в ЖТФ. 1979. Т. 5. В. 16. С. 1001-1006.

[7] Полищук В.П., Яриев И.М. // ТВТ. 1996. Т. 34. В. 3. С. 385 391.

[8] Musa G.S., Ehrich H., Schuhmann J. // IEEE Trans. Plasma Sci. 1997. V. 25. N 2. P. 386-391.

[9] Borisenko A.G., Saenko V.A., Rudnitsky V.A. // IEEE Trans. Plasma Sci. 1999. V. 27. N 4. P. 877-881.

[10] Borisenko A.G., Podzirei Yu.S. // Prob. Atom. Sci. Tech. 2015. N 1. Ser: Plasma Phys. (21). P. 177-180.

[11] Магунов А.Н. // Науч. приборостроение. 2010. Т. 20. № 3. C. 22-26. 